\title{
Súfilis congênita: uma análise epidemiológica no Estado de Minas Gerais
}

\author{
Congenital syphilis: an epidemiological analysis in the State of Minas Gerais \\ Sífilis congénita: un análisis epidemiológico en el Estado de Minas Gerais
}

Recebido: 22/09/2021 | Revisado: 29/09/2021 | Aceito: 08/10/2021 | Publicado: 11/10/2021

\author{
Marcele Soares Cortes Queiroz \\ ORCID: https://orcid.org/0000-0003-2736-8537 \\ Centro Universitário de Patos de Minas, Brasil \\ E-mail: marcelescq@unipam.edu.br \\ Gabriella Stéphany Brito Teixeira \\ ORCID: https://orcid.org/0000-0003-4042-5479 \\ Centro Universitário de Patos de Minas, Brasil \\ E-mail: gabriellastephany@unipam.edu.br \\ Luísa Macedo Nalin \\ ORCID: https://orcid.org/0000-0002-8667-1870 \\ Centro Universitário de Patos de Minas, Brasil \\ E-mail: luisamn@unipam.edu.br \\ Maria Isadora Nogueira \\ ORCID: https://orcid.org/0000-0003-1780-8236 \\ Centro Universitário de Patos de Minas, Brasil \\ E-mail: mariaisadora@unipam.edu.br \\ Priscila Capelari Orsolin \\ ORCID: https://orcid.org/0000-0001-7366-7437 \\ Centro Universitário de Patos de Minas, Brasil \\ E-mail: priscilaco@unipam.edu.br
}

\begin{abstract}
Resumo
Introdução: A sífilis é uma infecção sexualmente transmissível que tem como agente etiológico o Treponema Pallidum. A doença é um grave problema de saúde pública no Brasil devido à sua alta incidência em gestantes e a sua consequente transmissão para crianças em grande parte dos casos não tratados. Objetivo: abordar aspectos epidemiológicos relevantes ao estudo da sífilis congênita no estado de Minas Gerais, destacando o panorama epidemiológico entre os anos de 2010 e 2020. Metodologia: estudo quantitativo, descritivo, do tipo transversal. Por meio de dados do Sistema de Informação de Agravos e Notificação - SINAN- DATASUS, foram relacionados fatores, como taxa de incidência, idade da criança, diagnóstico final, óbitos, realização do pré-natal, momento do diagnóstico e esquema de tratamento. Para a construção das tabelas, foi utilizado o cálculo da incidência. Resultados: Os resultados evidenciaram que a grande maioria dos casos de sífilis não tratados em gestantes tem como consequência a sífilis congênita. Conclusão: Os dados permitem estabelecer relação entre o tratamento adequado da sífilis em gestantes e a prevenção da transmissão vertical. Portanto, a realização habitual do pré-natal, é capaz de minimizar possíveis complicações relacionadas a este agravo.
\end{abstract}

Palavras-chave: Sífilis congênita; Cuidado pré-natal; Epidemiologia descritiva; Registros públicos de dados de cuidados de saúde.

\begin{abstract}
Introduction: Syphilis is a sexually transmitted infection whose etiological agent is Treponema Pallidum. The disease is a serious public health problem in Brazil due to its high incidence in pregnant women and its consequent transmission to children in most untreated cases. Objective: to address epidemiological aspects relevant to the study of congenital syphilis in the state of Minas Gerais, highlighting the epidemiological panorama between 2010 and 2020. Methodology: quantitative, descriptive, cross-sectional study. Through data from the Disease Information and Notification System - SINANDATASUS, factors such as incidence rate, child's age, final diagnosis, deaths, prenatal care, time of diagnosis and treatment scheme were related. For the construction of the tables, the calculation of the incidence was used. Results: The results showed that the vast majority of cases of untreated syphilis in pregnant women have congenital syphilis as a consequence. Conclusion: The data allow us to establish a relationship between the adequate treatment of syphilis in pregnant women and the prevention of vertical transmission. Therefore, the usual performance of prenatal care is able to minimize possible complications related to this health problem.
\end{abstract}

Keywords: Congenital syphilis; Prenatal care; Descriptive epidemiology; Public records health care data.

\section{Resumen}

Introducción: La sífilis es una infección de transmisión sexual cuyo agente etiológico es Treponema pallidum. La enfermedad es un grave problema de salud pública en Brasil debido a su alta incidencia en mujeres embarazadas y su 
consecuente transmisión a los niños en la mayoría de los casos no tratados. Objetivo: abordar aspectos epidemiológicos relevantes para el estudio de la sífilis congénita en el estado de Minas Gerais, destacando el panorama epidemiológico entre 2010 y 2020. Metodología: estudio cuantitativo, descriptivo, transversal. A través de datos del Sistema de Información y Notificación de Enfermedades - SINANDATASUS, se relacionaron factores como tasa de incidencia, edad del niño, diagnóstico final, defunciones, atención prenatal, tiempo de diagnóstico y esquema de tratamiento. Para la construcción de las tablas se utilizó el cálculo de la incidencia. Resultados: Los resultados mostraron que la gran mayoría de los casos de sífilis no tratada en mujeres embarazadas tienen como consecuencia sífilis congénita. Conclusión: Los datos permiten establecer una relación entre el tratamiento adecuado de la sífilis en gestantes y la prevención de la transmisión vertical. Por tanto, la realización habitual de la atención prenatal es capaz de minimizar las posibles complicaciones relacionadas con este problema de salud.

Palabras clave: Sífilis congénita; Cuidado prenatal; Epidemiología descriptiva; Registros públicos de datos sanitarios.

\section{Introdução}

A sífilis é uma infecção sexualmente transmissível de caráter sistêmico e crônico com períodos de agudização, que é causada pela bactéria Treponema Pallidum. Tal patologia é conhecida como sífilis adquirida, quando é transmitida por via sexual no contato com as lesões contagiantes (Silva, Pereira, Aidar, Souza \& Costa, 2020).

A Sífilis congênita já seria o resultado da disseminação hematogênica do T. pallidum da mãe para o feto, predominantemente por via transplacentária, mas também pode ocorrer durante o parto e na amamentação, se houver contato do recém-nascido com lesões maternas. A Sífilis congênita pode ser classificada em precoce ou tardia. O primeiro tipo surge até o segundo ano de vida da criança e representa um acometimento de difícil diagnóstico, visto que mais da metade desses pacientes são assintomáticos ao nascimento ou possuem sinais e sintomas discretos e não específicos. Existem algumas manifestações clínicas, como prematuridade, baixo peso ao nascimento, hepatomegalia com ou sem esplenomegalia, lesões cutâneas, icterícia, anemia, dentre outras. Já o segundo tipo, Sífilis tardia, surge após o segundo ano de vida e tem como seus principais sinais e sintomas a tíbia em lâmina de sabre, articulações de Clutton, fronte "olímpica", nariz "em sela", molares “em amora”, mandíbula curta, surdez neurológica e dificuldade no aprendizado (Brasil, 2015).

Quando há sífilis na gestação, o risco de acometimento fetal pela doença costuma variar de 70 a $100 \%$, sendo que tal porcentagem varia dependendo da fase de infecção na gestante e do trimestre da gestação. Nesse sentido, torna-se extremamente importante e necessário realizar o teste para sífilis (VDRL, RPR) duas vezes durante a gestação, sendo uma na primeira consulta de pré-natal e outra durante o terceiro trimestre de gestação. Além disso, é preciso também realizar tal teste no momento de internação hospitalar (Brasil, 2012).

A sífilis em gestantes é uma doença de notificação compulsória no Brasil desde 2005 e, por isso, houve um aumento no número de casos diagnosticados, embora uma grande parte seja diagnosticada tardiamente. Assim, é possível observar que tal realidade acarreta consequências maternas e reflete uma quantidade considerável de casos de sífilis congênita não tratada que atinge os recém-nascidos. Nesse sentido, pode-se fazer uma relação com a sífilis congênita e com um pré-natal de baixa qualidade, o que vai contra as propostas da Rede Cegonha, implementada em 2011 (Costa, Santos, Silva, Barcelos \& Guerra, 2017).

De acordo com a Organização Mundial da Saúde (OMS), a sífilis congênita é uma das doenças mais graves que podem ser evitadas durante a gestação, quando o pré-natal oferecido é eficiente e o tratamento é feito de forma correta nas gestantes infectadas. É importante dizer que as altas taxas de transmissão vertical estão associadas a falhas na assistência, como início tardio do pré-natal, quebra na continuidade do cuidado com mudança de unidade de saúde, dificuldades no diagnóstico da sífilis durante a gestação, falhas no tratamento da gestante e do parceiro e falta de orientações acerca da doença e de sua prevenção (Costa et al., 2017).

Nesse sentido, essa pesquisa tem como objetivo descrever os aspectos de relevância no que tange os casos de sífilis congênita no estado de Minas Gerais, a partir de dados do DATASUS registrados no período de janeiro de 2010 a junho de 2020. A proposta integra uma breve abordagem sobre taxa de incidência, idade da criança, diagnóstico final, óbitos, realização 
do pré-natal, momento do diagnóstico e esquema de tratamento, buscando evidenciar o comportamento epidemiológico da distribuição dos casos.

\section{Metodologia}

O presente estudo é classificado como um estudo quantitativo, descritivo, do tipo transversal. O levantamento dos casos confirmados de Sífilis congênita foi realizado a partir de dados registrados no Sistema de Informação de Agravos e Notificação - SINAN- DATASUS, no estado de Minas Gerais. Foram considerados os dados referentes ao período de janeiro de 2010 a junho de 2020, sendo consideradas as variáveis: taxa de incidência, idade da criança no momento do diagnóstico, diagnóstico final, óbitos, realização do pré-natal, momento do diagnóstico e esquema de tratamento.

Posteriormente, os resultados foram colocados no Microsoft Excel, para tabulação dos dados presentes ao longo do artigo, em tabelas que apresentam números absolutos (n) e relativos (\%). As variáveis foram avaliadas de acordo com a distribuição ao longo dos anos. Não foi necessária a apreciação do Comitê de Ética em Pesquisa - CEP pelo fato de se tratar de dados públicos.

O trabalho foi realizado nas seguintes etapas: (I) pesquisa bibliográfica, (II) levantamento de dados acerca dos casos de sífilis congênita no estado de Minas Gerais no período de 2010 a junho de 2020 (III) e descrição dos dados encontrados.

\section{Resultados e Discussão}

A partir de uma busca nas bases de dados no DATASUS-TABNET foi possível estabelecer um quadro geral em torno da sífilis congênita no estado de Minas Gerais no período de janeiro de 2010 e junho de 2020. Desse modo, foram obtidos dados suficientes para traçar um perfil epidemiológico que pôde ser mesclado com o referencial teórico disponível.

No que se refere ao número de casos de sífilis diagnosticados no período considerado, os resultados do DATASUS mostram que houve uma elevada incidência de casos de sífilis congênita em menores de um ano de idade. Nos anos de 2018 e 2019 foram registrados 2.442 e 2.286 casos, respectivamente, nessa faixa etária; e houve um total de registros de 14.168 crianças nessa faixa etária com este agravo, considerando todo o período analisado. Além disso, o número de ocorrências aumentou progressivamente com o decorrer dos anos, visto que em 2010 foram registrados 228 casos e em 2019, 2.286. Em paralelo, no mesmo período analisado, os dados públicos mostraram que a incidência de sífilis em gestantes também apresentou crescimento exponencial, de modo que em 2010 ocorreram 362 casos, enquanto em 2019, 4.697 casos. O número total de gestantes com sífilis nesse período foi 25.603 , de modo que em mais da metade das pacientes houve transmissão da doença para seus filhos, seja durante a gestação ou após o parto.

Em grávidas não tratadas, a taxa de transmissão vertical pode alcançar valores acima de $80 \%$, por meio da bactéria ( $T$. pallidum) que atravessa a barreira placentária e chega ao concepto e, pode ocorrer também durante o parto e amamentação, caso haja contato da criança com lesões maternas (Silva et al., 2020; Costa et al., 2017). Relaciona-se a esse salto do número de casos, o comportamento de risco da população no que se refere principalmente à relação sexual desprotegida. O desenvolvimento de fármacos capazes de controlar e até mesmo tratar Infecções Sexualmente Transmissíveis (ISTs) e que podem aumentar a sobrevida dos infectados contribuiu para que os indivíduos se tornassem despreocupados com a prevenção dessas doenças (Costa et al., 2017). Em paralelo, a ampliação da testagem das gestantes, com maior disponibilidade de testes rápidos, preconizada pelo Ministério da Saúde durante o pré-natal, promove um aumento nas taxas de detecção de sífilis nas mulheres grávidas e, consequentemente, possibilita o rastreio da sífilis congênita, o que promove a elevação desses índices (Brasil, 2012).

No período analisado, grande parte dos casos de sífilis congênita em Minas Gerais foi detectada em filhos de mulheres que realizaram pré-natal, com uma incidência percentual de 85,9\% por ano, em média. Além disso, os anos com maiores índices foram 2018 e 2019, com 2.191 e 2.035 ocorrências respectivamente. Entre a distribuição total de casos, o número de 
casos de sífilis congênita entre os filhos de gestantes que não realizaram pré-natal foi expressivo, com 1.533 registros (Tabela $1)$.

Tabela 1. Distribuição absoluta (n) e percentual (\%) de casos de sífilis congênita segundo informação sobre realização de prénatal da mãe por ano de diagnóstico.

\begin{tabular}{llll}
\hline Ano & Realizou pré-natal n $(\%)$ & Não realizou pré-natal n $(\%)$ & Ignorado n $(\%)$ \\
\hline 2010 & $193(83,9)$ & $29(12,6)$ & $8(3,5)$ \\
2011 & $240(76,9)$ & $53(17)$ & $19(6,1)$ \\
2012 & $381(75)$ & $98(19,3)$ & $29(5,7)$ \\
2013 & $504(78,4)$ & $103(16)$ & $36(5,6)$ \\
2014 & $789(83,3)$ & $130(13,7)$ & $28(3)$ \\
2015 & $1199(84,3)$ & $147(10,3)$ & $63(4,3)$ \\
2016 & $1270(86,2)$ & $140(9,5)$ & $39(2,1)$ \\
2017 & $1600(88,2)$ & $176(9,7)$ & $42(1,7)$ \\
2018 & $2191(89,5)$ & $216(8,8)$ & $59(2,6)$ \\
2019 & $2035(88,5)$ & $206(9)$ & $35(4,1)$ \\
2020 & $747(87,9)$ & $68(8)$ & 482 \\
\hline
\end{tabular}

Fonte: SINAN - DATASUS (2020).

Isso reforça o caráter fundamental do pré-natal no que se refere à prevenção e tratamento precoce da sífilis congênita, evitando seus possíveis danos. Na atenção básica, o teste para verificar infecção por sífilis na gestante deve ser feito na primeira consulta do pré-natal, preferencialmente no primeiro trimestre, no início do terceiro trimestre e no momento do parto ou curetagem pós-aborto (Brasil, 2020). A sífilis congênita torna-se de fácil prevenção à medida que é diagnosticada precocemente. Dessa maneira, a partir da testagem adequada feita durante o pré-natal é determinado o tratamento às gestantes positivas a fim de minimizar os riscos à criança (Favero, Ribas, Costa \& Bonafé, 2019). Assim, o pré-natal realizado adequadamente serve como sentinela na busca de agravos para minimizar complicações tanto para as crianças quanto para as mães.

Segundo a análise realizada, o diagnóstico da sífilis congênita é dado, em mais da metade dos casos, durante o prénatal, seguido pelo momento do parto, em que, durante o período compreendido entre janeiro de 2010 e junho de 2020 , corresponde a 25,6\% dos diagnósticos efetuados. Por outro lado, a porcentagem de diagnósticos realizados após o parto ainda é considerável, representando 7,6\% do total (Tabela 2). Logo, é evidente a importância do pré-natal e da testagem para profilaxia e adesão ágil ao tratamento.

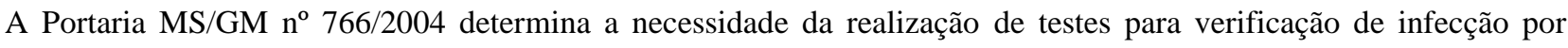
sífilis pelo menos duas vezes na gestação e no momento do parto ou curetagem pós-aborto (Brasil, 2012). Em concordância a essa determinação, no período analisado em Minas Gerais, e em consequência da maior adesão das mulheres ao acompanhamento pré-natal, a maioria dos casos de sífilis congênita foi diagnosticado durante esse período, com um percentual médio de 59,7\%, seguido pelo diagnóstico no momento do parto ou da curetagem. 
Tabela 2. Distribuição absoluta (n) e percentual (\%) de casos de sífilis congênita segundo o momento de diagnóstico da sífilis materna por ano de diagnóstico.

\begin{tabular}{|c|c|c|c|c|c|}
\hline Ano & $\begin{array}{l}\text { Durante o pré-natal } \\
\text { n }(\%)\end{array}$ & $\begin{array}{lcc}\text { No } & \text { momento } & \text { do } \\
\text { parto/curetagem } n(\%) & \end{array}$ & $\begin{array}{l}\text { Após o parto n } \\
(\%)\end{array}$ & $\begin{array}{l}\text { Não realizado } \\
\text { n }(\%)\end{array}$ & $\begin{array}{l}\text { Ignorado } \mathbf{n} \\
(\%)\end{array}$ \\
\hline 2010 & $129(56,1)$ & $62(27)$ & $28(12,2)$ & - & $11(4,8)$ \\
\hline 2011 & $153(49)$ & $97(31,1)$ & $49(15,7)$ & $1(0,3)$ & $12(3,8)$ \\
\hline 2012 & $233(45,9)$ & $184(36,2)$ & $65(12,8)$ & $1(0,2)$ & $25(4,9)$ \\
\hline 2013 & $345(53,7)$ & $198(30,8)$ & 77 (12) & $5(0,8)$ & $18(2,8)$ \\
\hline 2014 & $585(61,8)$ & $235(24,8)$ & $96(10,1)$ & $4(0,4)$ & $27(2,9)$ \\
\hline 2015 & $867(61)$ & $371(26,1)$ & $101(7,1)$ & $8(0,6)$ & $75(5,3)$ \\
\hline 2016 & $948(64,4)$ & $378(25,7)$ & $89(6)$ & $10(0,7)$ & $48(3,3)$ \\
\hline 2017 & $1166(64,2)$ & $489(26,9)$ & $100(5,5)$ & $14(0,8)$ & $46(2,5)$ \\
\hline 2018 & $1658(67,7)$ & $574(23,4)$ & $145(5,9)$ & $20(0,8)$ & $52(2,1)$ \\
\hline 2019 & $1604(69,7)$ & $490(21,3)$ & $126(5,5)$ & $16(0,7)$ & $64(2,8)$ \\
\hline 2020 & $540(63,5)$ & $199(23,4)$ & $65(7,6)$ & $6(0,7)$ & $40(4,7)$ \\
\hline Total & 8.503 & 3.461 & 1.026 & 92 & 439 \\
\hline
\end{tabular}

Fonte: SINAN - DATASUS (2020).

De acordo com os dados analisados, o diagnóstico de sífilis congênita em Minas Gerais ocorre, em sua maioria, em crianças com menos de 7 dias de vida. Assim, entre o período de janeiro de 2010 a junho de 2020, foram diagnosticadas 13.594 crianças em tal faixa etária com essa doença no estado. Observa-se também que a faixa etária de 5 a 12 anos teve a menor taxa de diagnóstico para sífilis congênita, com um total de 16 diagnosticados no mesmo período. É notado também que a idade no momento do diagnóstico foi ignorada em 12 casos (Tabela 3). 
Tabela 3. Distribuição absoluta (n) e percentual (\%) dos casos de sífilis congênita, segundo idade da criança por ano de diagnóstico.

\begin{tabular}{|c|c|c|c|c|c|c|c|}
\hline Ano & $\begin{array}{l}\text { Menos de } 7 \text { dias } n \\
(\%)\end{array}$ & $\begin{array}{l}7 \text { a } 27 \text { dias } n \\
(\%)\end{array}$ & $\begin{array}{l}28 \text { a } 364 \text { dias } n \\
(\%)\end{array}$ & $\begin{array}{l}1 \text { ano } n \\
(\%)\end{array}$ & $\begin{array}{l}2 \text { a } 4 \text { anos } n \\
(\%)\end{array}$ & $\begin{array}{l}5 \text { a } 12 \text { anos n } \\
(\%)\end{array}$ & $\begin{array}{l}\text { Ignorado } \mathbf{n} \\
(\%)\end{array}$ \\
\hline 2010 & $212(92,2)$ & $6(2,6)$ & $10(4,3)$ & - & $1(0,4)$ & $1(0,4)$ & - \\
\hline 2011 & $284(91)$ & $15(4,8)$ & $10(3,2)$ & $1(0,3)$ & - & $2(0,6)$ & - \\
\hline 2012 & $484(95,3)$ & $8(1,6)$ & $13(2,6)$ & $1(0,2)$ & $1(0,2)$ & $1(0,2)$ & - \\
\hline 2013 & $612(95,2)$ & $11(1,7)$ & $19(3)$ & $1(0,2)$ & - & - & - \\
\hline 2014 & $900(95)$ & $25(2,6)$ & $18(1,9)$ & $4(0,4)$ & - & - & - \\
\hline 2015 & $1358(95,5)$ & $28(2)$ & $30(2,1)$ & $3(0,2)$ & $2(0,1)$ & $1(0,1)$ & - \\
\hline 2016 & $1426(96,8)$ & $15(1)$ & $22(1,5)$ & $5(0,3)$ & $4(0,3)$ & $1(0,1)$ & - \\
\hline 2017 & $1746(96,2)$ & $28(1,5)$ & $33(1,8)$ & $5(0,3)$ & $2(0,1)$ & $1(0,1)$ & - \\
\hline 2018 & $2368(96,7)$ & $40(1,6)$ & $34(1,4)$ & $3(0,1)$ & $2(0,1)$ & $2(0,1)$ & - \\
\hline 2019 & $2235(97,2)$ & $20(0,9)$ & $31(1,3)$ & $7(0,3)$ & $4(0,2)$ & $3(0,1)$ & - \\
\hline 2020 & $827(97,3)$ & $11(1,3)$ & $9(1,1)$ & $1(0,1)$ & $1(0,1)$ & $1(0,1)$ & - \\
\hline Total & 13.594 & 286 & 288 & 36 & 24 & 16 & 12 \\
\hline
\end{tabular}

Fonte: SINAN - DATASUS (2020).

Durante o período analisado no estado de Minas Gerais, a maior parte dos casos de sífilis congênita foi identificada em crianças com menos de 7 dias de vida, com um percentual médio de 95,3\% identificados nessa faixa etária ao longo dos anos (Tabela 3). Paralelamente, a maior parte dos casos de sífilis congênita no estado foi classificada como sífilis congênita recente. Esse agravo é classificado como recente quando ocorre nos dois primeiros anos de vida da criança e tardio quando acontece após os dois anos de idade (Andrade, Magalhães, Moraes, Tresoldi \& Pereira, 2018).

A partir da observação dos dados obtidos do DATASUS entre janeiro de 2010 a junho de 2020, nota-se que o diagnóstico final dos casos de sífilis congênita em Minas Gerais ocorre principalmente na forma recente, no período dos dois primeiros anos de vida, com incidência de 13.370 crianças acometidas, com a porcentagem de 93,8\%. Dos 14.256 casos, apenas $0,5 \%$ foram considerados tardios. Além disso, 2,3\% manifestaram-se com aborto e 3,3\% foram natimortos.

O Ministério da Saúde preconiza a realização de teste não treponêmico a todos os recém-nascidos e suas mães, independente do histórico materno para sífilis, ainda em âmbito hospitalar, onde o parto foi realizado (Brasil, 2020). Tal fato contribui para que a maior parte dos casos de sífilis congênita seja diagnosticada nos primeiros dias de vida da criança e que são classificadas como sífilis recente, o que pode ser confirmado pela alta taxa de registro de casos de sífilis congênita recente somada ao grande número de notificações de casos de sífilis em crianças até os 7 dias de vida. 
Outro parâmetro analisado foi o número de óbitos por sífilis congênita em menores de um ano e o coeficiente bruto de mortalidade, segundo ano do óbito (Tabela 4). A partir dos dados, observa-se que os anos de 2015 e 2017 tiveram os maiores coeficientes de mortalidade, 8,6 e 8,4 , respectivamente.

Tabela 4. Distribuição absoluta (n) de óbitos por sífilis congênita em menores de um ano e coeficiente bruto de mortalidade (por 100.000 nascidos vivos) segundo ano do óbito.

\begin{tabular}{lll}
\hline Ano & Casos & Coeficiente \\
\hline 2010 & 1 & 0,4 \\
2011 & 5 & 1,9 \\
2012 & 1 & 0,4 \\
2013 & 4 & 1,5 \\
2014 & 6 & 2,2 \\
2015 & 23 & 8,6 \\
2016 & 7 & 2,8 \\
2017 & 22 & 8,4 \\
2018 & 21 & 8 \\
2019 & 14 & 5,3 \\
\hline
\end{tabular}

Fonte: SINAN - DATASUS (2020).

A sífilis congênita está entre as causas principais de abortos, natimortos e óbitos neonatais. Em Minas Gerais no período estudado, percebeu-se que ainda existem desfechos negativos para os casos de sífilis congênita (Tabela 4). De acordo com o SINAN, a maior parte dos casos de sífilis na gestação é diagnosticada tardiamente, a partir do segundo trimestre de gestação, de tal forma que há reflexo no maior percentual de transmissão vertical ao concepto de mulheres grávidas não tratadas, o que contribui com a manutenção dos índices de mortalidade e aborto nos últimos anos (Costa et al., 2017).

Além disso, outro fator preponderante para a manutenção dos coeficientes abordados acima seria a falha no tratamento da gestante, que envolve principalmente a falta ou inadequação do tratamento do parceiro, o qual ainda é pouco incluído nas consultas de pré-natal (Magalhães, Kawaguchi, Dias \& Calderon, 2013). O Ministério da Saúde recomenda a inclusão do parceiro na rotina pré-natal, principalmente para serem realizados os testes de triagem para verificação de possíveis doenças e agravos, sendo que no caso de IST's, o tratamento do parceiro é fundamental para a recuperação materna e para que a transmissão vertical possa ser evitada (Herrmann, Silva, Chakora \& Lima, 2016).

A taxa de transmissão vertical de sífilis, que pode ocorrer em qualquer fase da doença, atinge níveis altíssimos em mulheres grávidas infectadas não tratadas (Favero et al., 2019). No período analisado, no estado de Minas Gerais, grande parte dos casos de sífilis congênita recebeu esquema de tratamento inadequado, com uma média de 57,6\% (Tabela 5). 
Tabela 5. Distribuição absoluta (n) e percentual (\%) de casos de sífilis congênita segundo esquema de tratamento da mãe por ano de diagnóstico.

\begin{tabular}{|c|c|c|c|c|}
\hline Ano & Adequado n (\%) & Inadequado $\mathbf{n}(\%)$ & Não Realizado n (\%) & Ignorado n (\%) \\
\hline 2010 & $13(5,7)$ & $116(50,4)$ & $66(28,7)$ & $35(15,2)$ \\
\hline 2011 & $15(4,8)$ & $137(43,9)$ & $91(29,2)$ & $69(22,1)$ \\
\hline 2012 & $21(4,1)$ & $259(51)$ & $132(26)$ & $96(18,9)$ \\
\hline 2013 & $30(4,7)$ & $338(52,6)$ & $175(27,2)$ & $100(15,6)$ \\
\hline 2014 & $35(3,7)$ & $596(62,9)$ & $208(22)$ & $108(11,4)$ \\
\hline 2015 & $71(5)$ & $854(60,1)$ & $342(24,1)$ & $155(10,9)$ \\
\hline 2016 & $60(4,1)$ & $915(62,1)$ & $370(25,1)$ & $128(8,7)$ \\
\hline 2017 & $129(7,1)$ & $1108(61)$ & $460(25,3)$ & $118(6,5)$ \\
\hline 2018 & $140(5,7)$ & $1552(63,4)$ & $553(22,6)$ & $204(8,3)$ \\
\hline 2019 & $120(5,2)$ & $1377(59,9)$ & $536(23,3)$ & $267(11,6)$ \\
\hline 2020 & $58(6,8)$ & $501(58,9)$ & $206(24,2)$ & $85(10)$ \\
\hline Total & 916 & 8.215 & 3.461 & 1.664 \\
\hline
\end{tabular}

Fonte: SINAN - DATASUS (2020).

Outro estudo realizado em 2016, no estado do Paraná, mostrou dados semelhantes, sendo que em 53,7\% dos casos o tratamento das gestantes foi inadequado ou não realizado, de maneira similar ao observado nos dados referentes ao estado de Minas Gerais (Padovani, Oliveira \& Pelloso, 2018).

Quando o tratamento da sífilis gestacional é feito no primeiro trimestre de forma adequada com penicilina, costuma evitar a contaminação fetal (Figueiredo, Figueiredo, Souza, Tavares \& Vianna, 2020). Entretanto, em grande parte das ocorrências, o tratamento falha devido a utilização de doses inadequadas do antibiótico, especialmente nas fases de alta bacteremia, ou quando o tratamento ocorre no final da gestação, período em que as adaptações fisiológicas maternas, como aumento do débito cardíaco, da volemia e do fluxo sanguíneo renal, contribuem com alterações na farmacocinética da penicilina, com níveis subterapêuticos do medicamento no sangue (Guinsburg \& Santos, 2010).

\section{Conclusão}

Os resultados encontrados acerca do perfil epidemiológico da sífilis congênita no estado de Minas Gerais são condizentes com a preconização do Ministério da Saúde em relação à prevenção, ao diagnóstico e ao tratamento dessa patologia. A partir da análise dos dados constata-se que os motivos que exercem mais influência para que a doença evolua para sua forma mais complicada e para o óbito são o diagnóstico tardio e o tratamento inadequado. Assim, ressalta-se a importância da realização do acompanhamento pré-natal e do teste não treponêmico nas gestantes e nos recém-nascidos para a prevenção, além da educação sexual para evitar infecções sexualmente transmissíveis. Além disso, é fundamental a aplicação correta do tratamento precoce, com a antibioticoterapia, para a grávida e para o parceiro sexual, a fim de diminuir a incidência e a mortalidade da sífilis e de garantir o bem estar e o adequado desenvolvimento neuropsicomotor da criança. 
Como possibilidades para novas pesquisas referentes à sífilis congênita, sugerimos estudos comparativos entre os estados brasileiros, estabelecendo-se um panorama nacional acerca da doença, levando em consideração o potencial da rede pública de saúde em atender a população vulnerável a este agravo.

\section{Referências}

Andrade A. L. M. B., Magalhães P. V. S., Moraes M. M., Tresoldi A. T., \& Pereira R. M. (2018). Diagnóstico tardio de sífilis congênita: uma realidade na atenção à saúde da mulher e da criança no Brasil. Revista Paulista de Pediatria, 36(3), (pp. 376-381). <https://www.scielo.br/j/rpp/a/YW89sPHsznkK7m7fwvBFXJn/abstract/?lang=pt>

Ministério da Saúde. (2012). Cadernos de Atenção Básica no 32. <https://bvsms.saude.gov.br/bvs/publicacoes/cadernos_atencao_basica_32_prenatal.pdf>

Ministério da Saúde. (2012). Gestação de alto risco: manual técnico. [Manual] Departamento de Ações Programáticas Estratégicas.

Ministério da Saúde. (2020). Secretaria de Ciência, Tecnologia, Inovação e Insumos Estratégicos em Saúde. Departamento de Gestão e Incorporação de Tecnologias e Inovação em Saúde. Protocolo Clínico e Diretrizes Terapêuticas para Prevenção da Transmissão Vertical do HIV, Sífilis e Hepatites Virais. Recuperado em 14 maio, 2021, de <http://conitec.gov.br/images/Consultas/Relatorios/2020/PCDT_PTV_HIV_CP_42_2020.pdf>

Ministério da Saúde. (2015). Secretaria de Vigilância em Saúde. Departamento de DST, Aids e Hepatites Virais. Protocolo Clínico e Diretrizes Terapêuticas para Atenção Integral a as Pessoas com Sexualmente <https://bvsms.saude.gov.br/bvs/publicacoes/protocolo_clinico_diretrizes_terapeutica_atencao_integral_pessoas_infeccoes_sexualmente_transmissiveis.pdf>

Costa, C. V., Santos, I. A. B., Silva, J. M., Barcelos, T. F., \& Guerra, H. S. (2017). Sífilis congênita: repercussões e desafios. Arquivos Catarinenses de Medicina, 46(3), (pp. 194-202). <http://www.acm.org.br/acm/seer/index.php/arquivos/article/view/94>

Favero M. L. D. C., Ribas, K. A. W., Costa, M. C. D., \& Bonafé, S. M. (2019). Sífilis congênita e gestacional: notificação e assistência pré-natal. Archives Health Sciences, 26(1), (pp. 2-8). <https://www.cienciasdasaude.famerp.br/index.php/racs/article/view/1137>

Figueiredo D. C. M. M., Figueiredo A. M., Souza T. K. B., Tavares G., \& Vianna R. P. T. (2020). Relação entre oferta de diagnóstico e tratamento da sífilis na atenção básica sobre a incidência de sífilis gestacional e congênita. Cadernos de Saúde Pública, 36(3), e00074519. <https://www.scielo.br/j/csp/a/8syf4sN3Q5vZSw8mwk6zkDy/?lang=pt>

Guinsburg R., \& Santos A. M. N. (2010). Critérios Diagnósticos e Tratamento da Sífilis Congênita. Documento Científico - Departamento de Neonatologia Sociedade Brasileira de Pediatria. 〈https://www.sbp.com.br/fileadmin/user_upload/pdfs/tratamento_sifilis.pdf>

Herrmann A., Silva M. L., Chakora E. S., \& Lima D. C. (2016) Guia do Pré-Natal do Parceiro para Profissionais de Saúde. <https://portalarquivos2.saude.gov.br/images/pdf/2016/agosto/11/guia_PreNatal.pdf>

Magalhães D. M. S., Kawaguchi I. A. L., Dias A., \& Calderon I. M. P. (2013). Sífilis materna e congênita: ainda um desafio. Caderno de Saúde Pública, 29(6), (pp. 1109-1120). <https://www.scielo.br/j/csp/a/WM4wjfcJBy9Yb4FTvjhvCDz/?lang=pt\&format=pdf>

Ministério da Saúde. Departamento de Doenças de Condições Crônicas e Infecções Sexualmente Transmissíveis. (2020). <http://tabnet.datasus.gov.br/cgi/deftohtm.exe?sinannet/cnv/sifilismg.def>

Padovani C., Oliveira R. R., \& Pelloso S. M. (2018). Sífilis na gestação: associação das características maternas e perinatais em região do sul do Brasil. Revista Latino-Americana de Enfermagem, 26:e3019. <https://www.scielo.br/j/rlae/a/KXZGyqSjq4kVMvTL3sFP7zj/?format=pdf\&lang=pt>

Silva, M. F. C. F., Pereira, S. M. X., Aidar, T. P. S., Souza, R. G., \& Costa, R. F. C. (2020) Sífilis congênita como uma abordagem sistêmica. Brazilian Journal of Development, 6(7), (pp. 51840-51848). <https://www.brazilianjournals.com/index.php/BRJD/article/view/13969/11677> 